\title{
Tracking Controller Design for Diving Behavior of an Unmanned Underwater Vehicle
}

\author{
Yi-Hsiang Tseng, ${ }^{1,2}$ Chung-Cheng Chen, ${ }^{1}$ Chung-Huo Lin, ${ }^{1}$ and Yuh-Shyan Hwang ${ }^{2}$ \\ ${ }^{1}$ Department of Electrical Engineering, Hwa Hsia Institute of Technology, New Taipei City 235, Taiwan \\ ${ }^{2}$ Graduate Institute of Computer and Communication Engineering, National Taipei University of Technology, Taipei 10608, Taiwan
}

Correspondence should be addressed to Chung-Cheng Chen; ccc49827@ms25.hinet.net

Received 15 February 2013; Accepted 21 March 2013

Academic Editor: Chang-Hua Lien

Copyright (C) 2013 Yi-Hsiang Tseng et al. This is an open access article distributed under the Creative Commons Attribution License, which permits unrestricted use, distribution, and reproduction in any medium, provided the original work is properly cited.

The study has investigated the almost disturbance decoupling problem of nonlinear uncertain control systems via the fuzzy feedback linearization approach. The significant dedication of this paper is to organize a control algorithm such that the closed-loop system is active for given initial condition and bounded tracking trajectory with the input-to-state stability and almost disturbance decoupling performance. This study presents a feedback linearization controller for diving control of an unmanned underwater vehicle. Unmanned underwater vehicle proposes difficult control subject due to its nonlinear dynamics, uncertain models, and the existence of disturbances that are difficult to measure. In general, while investigating the diving dynamics of an unmanned underwater vehicle, the pitch angle is always assumed to be small. This assumption is a strong restricting constraint in many interesting practical applications and will be relaxed in this study.

\section{Introduction}

In the past three decades, the utilization of unmanned underwater vehicle (UUV) has rapidly developed due to the applications of them to operate in deeper and riskier environments where human divers cannot arrive as scientific inspection of the deep sea, underwater cave exploration, oceanographic mapping, exploitation of underwater resources, long range survey, and marine warfare $[1,2]$.

Tracking controller design for diving behavior of an UUV is difficult task in the investigation of appropriate algorithms for motion and position control because the UUV dynamics includes inherently hydrodynamics and inertial nonlinearities, modeling uncertainties, disturbances of varying drag forces, and the coupling problems between degrees of freedom. So the controller for UUV should be robust to suppress the uncertain effects from nonlinearity and error of modeling and the interferences from complicated external environment. Therefore, the traditional linear control approach cannot resolve the UUV appropriately after linearizing the UUV model for small range operation [3].

The diving behavior of an unmanned underwater vehicle has been approximated to a linear system [4-6] based on two traditional assumptions, and furthermore the traditional linear control schemes can be applied. One assumption is that the pitch angle is close to zero in the diving plane for maneuvering, and the other one is that the pitch motion dynamics can be approximated as a linear equation by using Taylor's expansion [1]. However, these two assumptions may create severe results in some practical applications due to their large modeling inaccuracy [7]. In this study, we directly solve the nonlinear dynamics of the depth motion without any restricting condition on the pitch angle of the UUV.

Recently, variable structure approach has been applied to treat nonlinear control system. However, inherited chattering effect may result in unmodeled high-frequency and even force system to be instable for variable structure approach structure. Adaptive backstepping approach has 
been an important tool for nonlinear strict-feedback systems and pure-feedback systems [8]. However, if the pitch angle is not close to zero, then the UUV's diving dynamics equation cannot be expressed in the strict-feedback form or some other specific form investigated in [9]. Therefore, it is challengeable to apply the adaptive backstepping approach directly to the depth control with large pitch angle. The output regulation control [10] is applied to the control system in which the output variables are assumed to be drived by an exosystem. However, the desired regulation problem needs to find out the troublesome solution of partial-differential equation and the problem of the transient tracking errors [11]. In general, the nonlinear $H^{\infty}$ control has to address the arduous nonlinear Hamilton-Jacobi equation [12]. Only for some specific control systems we can obtain a closed-form solution. The internalmodel-principle approach, addressing a first-order partialdifferential equation of the center manifold [13], transfers the tracking problem to nonlinear output regulation form. Only for some particular control systems and desired trajectories, the asymptotic solutions can be obtained [14]. The $H^{\infty}$ adaptive fuzzy control has been applied to systematically investigate some control systems [15]. Its shortcoming is that the complicated parameter update law makes it be impractical. During the past decade, the feedback linearization approach has been the research direction for nonlinear systems $[16,17]$ and has been utilized successfully to solve many practical applications including the Three-Phase Photovoltaic Inverter [18], ball and beam system [16], and holonomic constrained robotic systems [19].

Fuzzy logic approach has been utilized to many industrial processes $[20,21]$. Its designing structure is summarized as follows. First express the original fuzzy system as the TakagiSugeno model with fuzzy defined regions where the original system is locally linearized. The desired control design of resulting augmented system is organized by a combination of linear controllers constructed for each separated local linear part of the fuzzy model based on the parallel distributed compensation technique $[22,23]$. For the stability investigation of fuzzy system, many researches are addressed [24, 25]. The stability analysis of fuzzy system can be mainly focused on Tanaka-Sugeno's theorem. However, it is troublesome to carry out the common positive definite matrix $P$ for linear matrix inequality approach [26] even if $P$ is a second-order model. To solve the shortcoming of calculating the modelmatrix $P$ for fuzzy logic approach, we will put forward a new controller design to achieve the almost disturbance decoupling property and the overall system is stabilized. Our proposed designing process is summarized as follows. First, a tracking control is constructed in order to achieve the almost disturbance decoupling performance and the stability of the closed-loop system response within a tunable global final attractor via the feedback linearization method. Once the tracking trajectories are steered to touch the global final attractor with the given radius, the fuzzy logic control instantly is added to achieve the desired convergence rate. In order to demonstrate the interesting industrial application, this study has favorably designed the almost disturbance decoupling controller for an unmanned underwater vehicle.

\section{Controller Design}

2.1. Controller Design for Feedback Linearization Approach. We will investigate the following nonlinear uncertain control system with bounded time-varying noises:

$$
\begin{aligned}
{\left[\begin{array}{c}
\dot{x}_{1} \\
\dot{x}_{2} \\
\vdots \\
\dot{x}_{n}
\end{array}\right]=} & {\left[\begin{array}{c}
f_{1}\left(x_{1}, x_{2}, \ldots, x_{n}\right) \\
f_{2}\left(x_{1}, x_{2}, \ldots, x_{n}\right) \\
\vdots \\
f_{n}\left(x_{1}, x_{2}, \ldots, x_{n}\right)
\end{array}\right]+\left[\begin{array}{c}
g_{1}\left(x_{1}, x_{2}, \ldots, x_{n}\right) \\
g_{2}\left(x_{1}, x_{2}, \ldots, x_{n}\right) \\
\vdots \\
g_{n}\left(x_{1}, x_{2}, \ldots, x_{n}\right)
\end{array}\right] u } \\
& +\left[\begin{array}{c}
\Delta f_{1} \\
\Delta f_{2} \\
\vdots \\
\Delta f_{n}
\end{array}\right]+\sum_{i=1}^{p} q_{i}^{*} \theta_{i d} \\
\equiv & f(X(t))+g(X(t)) u+\Delta f+\sum_{i=1}^{p} q_{i}^{*} \theta_{i d},
\end{aligned}
$$

where $X(t) \equiv\left[\begin{array}{llll}x_{1}(t) & x_{2}(t) & \cdots & x_{n}(t)\end{array}\right]^{T} \in \mathfrak{R}^{n}$ is the system state vector, $u \in \mathfrak{R}^{1}$ is the input variable, $y \in \mathfrak{R}^{1}$ is the output variable, $\theta_{d} \equiv\left[\begin{array}{llll}\theta_{1 d}(t) & \theta_{2 d}(t) & \cdots & \theta_{p d}(t)\end{array}\right]^{T}$ is a bounded time-varying noise vector, and $\Delta f \equiv\left[\begin{array}{llll}\Delta f_{1} & \Delta f_{2} & \cdots & \Delta f_{n}\end{array}\right]^{T} \epsilon$ $\Re^{n}$ is unknown function denoting as $\Delta f=\sum_{i=1}^{p} q_{i}^{*} \theta_{i u}$, where $\theta_{u} \equiv\left[\begin{array}{llll}\theta_{1 u}(t) & \theta_{2 u}(t) & \cdots & \theta_{p u}(t)\end{array}\right]^{T}$ is a bounded time-varying uncertainty. $f, g, q_{1}^{*}, \ldots, q_{p}^{*}$ are smooth vector fields on $\mathfrak{R}^{n}$ and $h(X(t)) \in \mathfrak{R}^{1}$ is a smooth function. The nominal system of original system is then described as follows:

$$
\begin{gathered}
\dot{X}(t)=f(X(t))+g(X(t)) u, \\
y(t)=h(X(t)) .
\end{gathered}
$$

The relative degree $r$ [27] is defined $1 \leq r<\infty$ such that

$$
\begin{gathered}
L_{g} L_{f}^{k} h(X(t))=0, \quad k<r-1, \\
L_{g} L_{f}^{r-1} h(X(t)) \neq 0
\end{gathered}
$$

for all $X \in \mathfrak{R}^{n}$ and $t \in[0, \infty)$, where the operator $L$ is denoted as the Lie derivative [28]. The desired trajectory $y_{t}(t)$ and its first $r$ derivatives are all uniformly bounded and

$$
\left\|\left[y_{t}(t), y_{t}^{(1)}(t), \ldots, y_{t}^{(r)}(t)\right]\right\| \leq \bar{O}
$$

where $\bar{O}$ is a positive real variable.

Reference [28] has exploited the fact that the transformation

$$
\Phi: \mathfrak{R}^{n} \longrightarrow \mathfrak{R}^{n}
$$

denoted as

$$
\begin{gathered}
\Phi_{i}(X(t)) \equiv \xi_{i}(t)=L_{f}^{i-1} h(X(t)), \quad i=1,2, \ldots, r \\
\Phi_{k}(X(t)) \equiv \eta_{k}(t), \quad k=r+1, r+2, \ldots, n
\end{gathered}
$$


and satisfying

$$
L_{g} \Phi_{k}(X(t))=0, \quad k=r+1, r+2, \ldots, n
$$

is a diffeomorphism based on the assumption of well-defined relative degree. For the sake of simplification, denote the tracking error to be

$$
\begin{gathered}
e_{i}(t) \equiv \xi_{i}(t)-y_{t}^{(i-1)}(t), \quad i=1,2, \ldots, r, \\
e(t) \equiv\left[\begin{array}{llll}
e_{1}(t) & e_{2}(t) & \cdots & e_{r}(t)
\end{array}\right]^{T} \in \mathfrak{R}^{r}
\end{gathered}
$$

and the tracking error multiplied with an adjustable positive real variable $\sigma$ to be

$$
\begin{gathered}
\overline{e_{i}}(t) \equiv \sigma^{i-1} e_{i}(t), \quad i=1,2, \ldots, r, \\
\bar{e}(t) \equiv\left[\begin{array}{llll}
\overline{e_{1}}(t) & \overline{e_{2}}(t) & \cdots & \overline{e_{r}}(t)
\end{array}\right]^{T} \in \mathfrak{R}^{r}, \\
\xi(t) \equiv\left[\begin{array}{llll}
\xi_{1}(t) & \xi_{2}(t) & \cdots & \xi_{r}(t)
\end{array}\right]^{T} \in \mathfrak{R}^{r}, \\
\eta(t) \equiv\left[\begin{array}{llll}
\eta_{r+1}(t) & \eta_{r+2}(t) & \cdots & \eta_{n}(t)
\end{array}\right]^{T} \in \mathfrak{R}^{n-r}, \\
q(\xi(t), \eta(t)) \\
\equiv\left[\begin{array}{llll}
L_{f} \Phi_{r+1}(t) & L_{f} \Phi_{r+2}(t) & \cdots & L_{f} \Phi_{n}(t)
\end{array}\right]^{T}, \\
\equiv\left[\begin{array}{llll}
q_{r+1} & q_{r+2} & \cdots & q_{n}
\end{array}\right]^{T} .
\end{gathered}
$$

Denote the desired phase-variable canonical matrix $A_{\mathrm{pvc}}$ to be

$$
A_{\mathrm{pvc}} \equiv\left[\begin{array}{ccccc}
0 & 1 & 0 & \cdots & 0 \\
0 & 0 & 1 & \cdots & 0 \\
& \vdots & & & \vdots \\
0 & 0 & 0 & \cdots & 1 \\
-n_{1} & -n_{2} & -n_{3} & \cdots & -n_{r}
\end{array}\right]_{r \times r} \text {, }
$$

where $n_{1}, n_{2}, \ldots, n_{r}$ are adjustable real variables such that $A_{\mathrm{pvc}}$ is Hurwitz matrix and the vector $B$ to be

$$
B \equiv\left[\begin{array}{lllll}
0 & 0 & \cdots & 0 & 1
\end{array}\right]_{r \times 1}^{T} .
$$

Define $P_{\mathrm{pd}}$ to be the matrix of the famous Lyapunov equation

$$
\begin{gathered}
A_{\mathrm{pvc}}^{T} P_{\mathrm{pd}}+P_{\mathrm{pd}} A_{\mathrm{pvc}}=-I, \\
\lambda_{\max } \equiv \text { the maximum eigenvalue of } \\
\text { the positive definite matrix } P_{\mathrm{pd}}, \\
\lambda_{\text {min }} \equiv \text { the minimum eigenvalue of } \\
\text { the positive definite matrix } P_{\mathrm{pd}} .
\end{gathered}
$$

Assumption 1. For all $t \geq 0, \eta \in \mathfrak{R}^{n-r}$ and $\xi \in \mathfrak{R}^{r}$, there exists a positive real variable $L_{k}$ such that the following property holds:

$$
\left\|q_{22}(\eta, \bar{e})-q_{22}(\eta, 0)\right\| \leq L_{k}(\|\bar{e}\|),
$$

where $q_{22}(\eta, \bar{e}) \equiv q(\xi, \eta)$.
For the sake of simplification, denote

$$
\begin{gathered}
u_{d} \equiv L_{g} L_{f}^{r-1} h(X(t)), \\
u_{c} \equiv L_{f}^{r} h(X(t)),
\end{gathered}
$$

$$
\overline{\bar{e}}=n_{1} \overline{e_{1}}+n_{2} \overline{e_{2}}+\cdots+n_{r} \overline{e_{r}} .
$$

Definition 2 (see [29]). Consider the control system $\dot{x}=$ $f(t, x, \theta)$, where $f:[0, \infty) \times \mathfrak{R}^{n} \times \mathfrak{R}^{n} \rightarrow \mathfrak{R}^{n}$ is a piecewise continuous function and has the locally Lipschitz property in $x$ and $\theta$. This control system is described to be input-to-state stable if there exists a class $K L$ function $\beta$, a class $K$ function $\gamma$, and positive real variables $k_{1}$ and $k_{2}$ such that for given initial state $x\left(t_{0}\right)$ with $\left\|x\left(t_{0}\right)\right\|<k_{1}$ and the system input $\theta(t)$ with $\sup _{t \geq t_{0}}\|\theta(t)\|<k_{2}$, the system states satisfy

$$
\|x(t)\| \leq \beta\left(\left\|x\left(t_{0}\right)\right\|, t-t_{0}\right)+\gamma\left(\sup _{t_{0} \leq \tau \leq t}\|\theta(\tau)\|\right)
$$

for all $t \geq t_{0} \geq 0$. Now we exploit the almost disturbance decoupling property as follows.

Definition 3 (see [30]). The tracking problem with almost disturbance decoupling property is defined to be globally solvable by the controller $u$ for the tracking-error system by a global diffeomorphism (5), if the controller $u$ possesses the following properties.

(i) The stystem is input-to-state stable with respect to disturbance inputs.

(ii) For any given initial value $\bar{x}_{e 0}:=\left[\begin{array}{ll}\bar{e}\left(t_{0}\right) & \eta\left(t_{0}\right)\end{array}\right]^{T}$, for any $t \geq t_{0}$, and for any $t_{0} \geq 0$,

$$
\begin{aligned}
& \left|y(t)-y_{d}(t)\right| \\
& \leq \beta_{11}\left(\left\|x\left(t_{0}\right)\right\|, t-t_{0}\right) \\
& \quad+\frac{1}{\sqrt{\beta_{22}}} \beta_{33}\left(\sup _{t_{0} \leq \tau \leq t}\|\theta(\tau)\|\right), \\
& \int_{t_{0}}^{t}\left[y(\tau)-y_{d}(\tau)\right]^{2} d \tau \\
& \leq \frac{1}{\beta_{44}}\left[\beta_{55}\left(\left\|\bar{x}_{e 0}\right\|\right)+\int_{t_{0}}^{t} \beta_{33}\left(\|\theta(\tau)\|^{2}\right) d \tau\right],
\end{aligned}
$$

where $\beta_{22}, \beta_{44}$ are some positive real variables, $\beta_{33}, \beta_{55}$ are class $K$ functions, and $\beta_{11}$ is a class $K L$ function.

Theorem 4. The tracking problem with almost disturbance decoupling performance for system (1a)-(1b) is globally solvable by the controller $u$ denoted by 


$$
\begin{aligned}
& {\left[L_{g} L_{f}^{r-1} h(X(t))\right]^{-1}} \\
& \times\left\{-L_{f}^{r} h(X)+y_{t}^{(r)}-\sigma^{-r} n_{1}\left[L_{f}^{0} h(X)-y_{t}\right]\right. \\
& \quad-\sigma^{1-r} n_{2}\left[L_{f}^{1} h(X)-y_{t}^{(1)}\right] \\
& \left.\quad-\cdots-\sigma^{-1} n_{r}\left[L_{f}^{r-1} h(X)-y_{t}^{(r-1)}\right]\right\}
\end{aligned}
$$

and the influence of disturbances on the tracking error can be arbitrarily attenuated by adjusting the real variable $\mathrm{N}_{2}$ :

$$
\begin{aligned}
& N_{2} \equiv \min \left\{m_{11}, m_{22}\right\} \text {, } \\
& m_{11} \equiv \delta-\left(2 \delta\left\|\Phi_{\xi}\right\|\left\|P_{p d}\right\|\right)^{2}-0.25 \text {, } \\
& m_{22} \equiv \mu m_{3}-\left(\mu L_{k} m_{4}\right)^{2}-\left(\mu L_{k} m_{4}\left\|\Phi_{\eta}\right\|\right)^{2} \text {, } \\
& \Phi_{\xi}(\sigma) \equiv\left[\begin{array}{ccc}
\sigma \frac{\partial}{\partial X} h q_{1}^{*} & \cdots & \sigma \frac{\partial}{\partial X} h q_{p}^{*} \\
\vdots & & \vdots \\
\sigma^{r} \frac{\partial}{\partial X} L_{f}^{r-1} h q_{1}^{*} & \cdots & \sigma^{r} \frac{\partial}{\partial X} L_{f}^{r-1} h q_{q}^{*}
\end{array}\right], \\
& \Phi_{\eta}(\sigma) \equiv\left[\begin{array}{ccc}
\frac{\partial}{\partial X} \Phi_{r+1} q_{1}^{*} & \cdots & \frac{\partial}{\partial X} \Phi_{r+1} q_{p}^{*} \\
\vdots & & \vdots \\
\frac{\partial}{\partial X} \Phi_{n} q_{1}^{*} & \cdots & \frac{\partial}{\partial X} \Phi_{n} q_{q}^{*}
\end{array}\right],
\end{aligned}
$$

where $\delta$ and $\mu$ are adjustable real variables, based on the fact that there exists a continuously differentiable function $V_{0}$ : $\mathfrak{R}^{n-r} \rightarrow \Re^{+}$such that the following properties hold for all $\eta \in \mathfrak{R}^{n-r}:$

(a) $m_{1}\|\eta\|^{2} \leq V_{0}(\eta) \leq m_{2}\|\eta\|^{2}, \quad m_{1}, m_{2}>0$,

(b) $\left(\nabla_{\eta} V_{0}\right)^{T} q_{22}(\eta, 0) \leq-m_{3}\|\eta\|^{2}, \quad m_{3}>0$,

(c) $\left\|\nabla_{\eta} V_{0}\right\| \leq m_{4}\|\eta\|, \quad m_{4}>0$.

Moreover, the overall tracking error is fascinated into a ball $B_{\underline{r}}, \underline{r}=\sqrt{N_{1} / N_{2}}$, with an convergence rate denoted by

$$
\begin{gathered}
\frac{1}{2}\left(\frac{N_{2}}{\omega_{2}}-\frac{N_{1}}{\omega_{2} \underline{r}^{2}}\right) \equiv \frac{1}{2} \alpha^{*}, \\
N_{1} \equiv \frac{1}{2}\left(\sup _{t_{0} \leq \tau \leq t}\left\|\theta_{d}(\tau)+\theta_{u}(\tau)\right\|\right)^{2} .
\end{gathered}
$$

Proof. Applying the coordinate transformation (5) results in

$$
\begin{aligned}
\dot{\xi}_{1}(t)= & \xi_{2}(t)+\frac{\partial h(X)}{\partial X} \sum_{i=1}^{p} q_{i}^{*}\left(\theta_{i d}+\theta_{i u}\right) \\
& \vdots \\
\dot{\xi}_{r-1}(t)= & \xi_{r}(t) \\
& +\frac{\partial L_{f}^{r-2} h(X(t))}{\partial X} \sum_{i=1}^{p} q_{i}^{*}\left(\theta_{i d}+\theta_{i u}\right) \\
\dot{\xi}_{r}(t)= & L_{f}^{r} h(X)+L_{g} L_{f}^{r-1} h(X) u \\
& +\sum_{i=1}^{p} \frac{\partial L_{f}^{r-1} h(X(t))}{\partial X} q_{i}^{*}\left(\theta_{i d}+\theta_{i u}\right) \\
\dot{\eta}_{k}(t)= & L_{f} \Phi_{k}+\sum_{i=1}^{p} \frac{\partial \Phi_{k}(X)}{\partial X} q_{i}^{*}\left(\theta_{i d}+\theta_{i u}\right) .
\end{aligned}
$$

Since

$$
\begin{gathered}
u_{c}(\xi(t), \eta(t)) \equiv L_{f}^{r} h(X(t)), \\
u_{d}(\xi(t), \eta(t)) \equiv L_{g} L_{f}^{r-1} h(X(t)), \\
q_{k}(\xi(t), \eta(t))=L_{f} \Phi_{k}(X), \quad k=r+1, r+2, \ldots, n ;
\end{gathered}
$$

the state and ouput equations of system (1a)-(1b) can be rewritten as follows:

$$
\begin{gathered}
\dot{\xi}_{i}(t)=\xi_{i+1}(t) \\
+\sum_{i=1}^{p} \frac{\partial}{\partial X} L_{f}^{i-1} h q_{i}^{*}\left(\theta_{i d}+\theta_{i u}\right), \quad i=1,2, \ldots, r-1, \\
\dot{\xi}_{r}(t)=u_{c}(\xi(t), \eta(t)) \\
+u_{d}(\xi(t), \eta(t)) u \\
+\sum_{i=1}^{p} \frac{\partial}{\partial X} L_{f}^{r-1} h q_{i}^{*}\left(\theta_{i d}+\theta_{i u}\right), \\
\dot{\eta}_{k}(t)=q_{k}(\xi(t), \eta(t)) \\
+\sum_{i=1}^{p} \frac{\partial}{\partial X} \Phi_{k}(X) q_{i}^{*}\left(\theta_{i d}+\theta_{i u}\right), \quad k=r+1, \ldots, n, \\
y(t)=\xi_{1}(t) .
\end{gathered}
$$

Denote

$$
\begin{aligned}
v \equiv & y_{t}^{(r)}-\sigma^{-r} n_{1}\left[L_{f}^{0} h(X)-y_{t}\right] \\
& -\sigma^{1-r} n_{2}\left[L_{f}^{1} h(X)-y_{t}^{(1)}\right] \\
& -\cdots-\sigma^{-1} n_{r}\left[L_{f}^{r-1} h(X)-y_{t}^{(r-1)}\right] .
\end{aligned}
$$


Utilizing (6), (9), (25), and (26) yields the controller as

$$
u=u_{d}^{-1}\left[-u_{c}+v\right] \text {. }
$$

The dynamic equations of system (1a)-(1b) can be shown as follows by substituting equation (30) into (2.35):

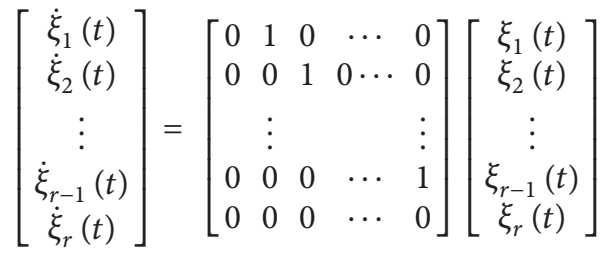$$
+\left[\begin{array}{c}
0 \\
0 \\
\vdots \\
0 \\
1
\end{array}\right] v
$$

$$
+\left[\begin{array}{c}
+\sum_{i=1}^{p} \frac{\partial}{\partial X} h q_{i}^{*}\left(\theta_{i d}+\theta_{i u}\right) \\
+\sum_{i=1}^{p} \frac{\partial}{\partial X} L_{f}^{1} h q_{i}^{*}\left(\theta_{i d}+\theta_{i u}\right) \\
\vdots \\
+\sum_{i=1}^{p} \frac{\partial}{\partial X} L_{f}^{r-1} h q_{i}^{*}\left(\theta_{i d}+\theta_{i u}\right)
\end{array}\right]
$$

$$
\left[\begin{array}{c}
\dot{\eta}_{r+1}(t) \\
\dot{\eta}_{r+2}(t) \\
\vdots \\
\dot{\eta}_{n-1}(t) \\
\dot{\eta}_{n}(t)
\end{array}\right]=\left[\begin{array}{c}
q_{r+1}(t) \\
q_{r+2}(t) \\
\vdots \\
q_{n-1}(t) \\
q_{n}(t)
\end{array}\right]+\left[\begin{array}{c}
\sum_{i=1}^{p} \frac{\partial}{\partial X} \Phi_{r+1} q_{i}^{*}\left(\theta_{i d}+\theta_{i u}\right) \\
\sum_{i=1}^{p} \frac{\partial}{\partial X} \Phi_{r+2} q_{i}^{*}\left(\theta_{i d}+\theta_{i u}\right) \\
\vdots \\
\sum_{i=1}^{p} \frac{\partial}{\partial X} \Phi_{n-1} q_{i}^{*}\left(\theta_{i d}+\theta_{i u}\right) \\
\sum_{i=1}^{p} \frac{\partial}{\partial X} \Phi_{n} q_{i}^{*}\left(\theta_{i d}+\theta_{i u}\right)
\end{array}\right]
$$

$$
y=\left[\begin{array}{lllll}
1 & 0 & \cdots & 0 & 0
\end{array}\right]_{r \times 1}\left[\begin{array}{c}
\xi_{1}(t) \\
\xi_{2}(t) \\
\vdots \\
\xi_{r-1}(t) \\
\xi_{r}(t)
\end{array}\right]_{r \times 1}=\xi_{1}(t) .
$$

Combining equations (9), (11), (14), and (29) verifies the fact that equations (31)-(33) can be rewritten into the following equations:

$$
\begin{gathered}
\dot{\eta}(t)=q(\xi(t), \eta(t))+\Phi_{\eta}\left(\theta_{d}+\theta_{u}\right) \\
\equiv q_{22}(\eta(t), \bar{e})+\Phi_{\eta}\left(\theta_{d}+\theta_{u}\right), \\
\sigma \bar{e}(t)=A_{\mathrm{pvc}} \bar{e}+\Phi_{\xi}\left(\theta_{d}+\theta_{u}\right),
\end{gathered}
$$

$$
y(t)=\xi_{1}(t) .
$$

Define $V(\bar{e}, \eta)$ by a weighted sum of $V_{0}(\eta)$ and $V_{1}(\bar{e})$,

$$
V(\bar{e}, \eta) \equiv V_{1}(\bar{e})+\mu V_{0}(\eta)
$$

as a composite Lyapunov function of the systems (34a) and (34b) [31], where $V_{1}(\bar{e})$ satisfies

$$
V_{1}(\bar{e}) \equiv \sigma \delta \bar{e}^{T} P_{\mathrm{pd}} \bar{e}
$$

where $\delta$ and $\mu$ are adjusting positive real variables. Utilizing (17)-(19), (21) and (23a), (23b), (23c), (23d), and (23e) yields the derivative of $V(\bar{e}, \eta)$ along the trajectories of $(34 \mathrm{a})$ and $(34 \mathrm{~b})$ as

$$
\begin{aligned}
\dot{V}_{1}= & \delta(\sigma \dot{\bar{e}})^{T} P_{\mathrm{pd}} \bar{e}+\delta \bar{e}^{T} P_{\mathrm{pd}}(\sigma \dot{\bar{e}}) \\
= & \delta\left\{A_{c} \bar{e}+\Phi_{\xi}\left(\theta_{d}+\theta_{u}\right)\right\}^{T} P_{\mathrm{pd}} \bar{e} \\
& +\delta \bar{e}^{T} P_{\mathrm{pd}}\left\{A_{c} \bar{e}+\Phi_{\xi}\left(\theta_{d}+\theta_{u}\right)\right\} \\
= & \delta\left\{\bar{e}^{T}\left(A_{\mathrm{pvc}}^{T} P_{\mathrm{pd}}+P_{\mathrm{pd}} A_{\mathrm{pvc}}\right) \bar{e}\right\} \\
& +\delta\left\{2\left(\theta_{d}+\theta_{u}\right)^{T} \Phi_{\xi}^{T} P_{\mathrm{pd}} \bar{e}\right\} \\
\leq & -\delta\|\vec{e}\|^{2}+2 \delta\left\|\left(\theta_{d}+\theta_{u}\right)\right\|\left\|\Phi_{\xi}\right\|\left\|P_{\mathrm{pd}}\right\|\|\vec{e}\| \\
\leq & -\delta\|\vec{e}\|^{2}+2 \delta\left\|\left(\theta_{d}+\theta_{u}\right)\right\|\left\|\Phi_{\xi}\right\|\left\|P_{\mathrm{pd}}\right\|\|\vec{e}\| \\
\leq & -\delta\|\vec{e}\|^{2}+\left(2 \delta\left\|\Phi_{\xi}\right\|\left\|P_{\mathrm{pd}}\right\|\right)^{2}\|\vec{e}\|^{2}+\frac{1}{4}\left\|\left(\theta_{d}+\theta_{u}\right)\right\|^{2},
\end{aligned}
$$

that is,

$$
\begin{aligned}
\dot{V}_{1} \leq & -\|\vec{e}\|^{2}\left[\delta-\left(2 \delta\left\|\Phi_{\xi}\right\|\left\|P_{\mathrm{pd}}\right\|\right)^{2}\right] \\
& +\frac{1}{4}\left\|\left(\theta_{d}+\theta_{u}\right)\right\|^{2} \\
\mu \dot{V}_{0}= & \mu\left(\frac{\partial V_{0}}{\partial \eta}\right)^{T} \dot{\eta}=\mu\left(\frac{\partial V_{0}}{\partial \eta}\right)^{T} \\
& \times\left[q_{22}(\eta, \bar{e})+\Phi_{\eta} \theta-q_{22}(\eta, 0)+q_{22}(\eta, 0)\right] \\
\leq & \mu\left\|\frac{\partial V_{0}}{\partial \eta}\right\|\left\|q_{22}(\eta, \bar{e})-q_{22}(\eta, 0)\right\| \\
& +\mu\left(\frac{\partial V_{0}}{\partial \eta}\right)^{T} q_{22}(\eta, 0)+\mu\left\|\frac{\partial V_{0}}{\partial \eta}\right\|\left\|\Phi_{\eta}\right\|\left\|\left(\theta_{d}+\theta_{u}\right)\right\| \\
\leq & \mu m_{4} L_{k}\|\eta\|\|\bar{e}\|-\mu m_{3}\|\eta\|^{2} \\
& +\mu m_{4}\left\|\Phi_{\eta}\right\|\|\eta\|\left\|\left(\theta_{d}+\theta_{u}\right)\right\| \\
\leq & \left(\mu m_{4} L_{k}\right)^{2}\|\eta\|^{2}+\frac{1}{4}\|\bar{e}\|^{2}
\end{aligned}
$$




$$
\begin{aligned}
& -\mu m_{3}\|\eta\|^{2}+\left(\mu m_{4}\left\|\Phi_{\eta}\right\|\right)^{2}\|\eta\|^{2} \\
& +\frac{1}{4}\left\|\left(\theta_{d}+\theta_{u}\right)\right\|^{2}
\end{aligned}
$$

that is,

$$
\begin{aligned}
\mu \dot{V}_{0} \leq & \|\eta\|^{2}\left[-\mu m_{3}+\left(\mu m_{4} L_{k}\right)^{2}+\left(\mu m_{4}\left\|\Phi_{\eta}\right\|\right)^{2}\right] \\
+ & \frac{1}{4}\|\bar{e}\|^{2}+\frac{1}{4}\left\|\left(\theta_{d}+\theta_{u}\right)\right\|^{2} .
\end{aligned}
$$

Therefore,

$$
\begin{aligned}
\dot{V}= & \dot{V}_{1}+\mu \dot{V}_{0} \\
\leq & -\|\vec{e}\|^{2}\left[\delta-\left(2 \delta\left\|\Phi_{\xi}\right\|\left\|P_{\mathrm{pd}}\right\|\right)^{2}-\frac{1}{4}\right] \\
& -\|\eta\|^{2}\left[\mu m_{3}-\left(\mu m_{4} L_{k}\right)^{2}-\left(\mu m_{4}\left\|\Phi_{\eta}\right\|\right)^{2}\right] \\
& +\frac{1}{2}\left\|\left(\theta_{d}+\theta_{u}\right)\right\|^{2} \\
\leq & -m_{11}\|\vec{e}\|^{2}-m_{22}\|\eta\|^{2}+\frac{1}{2}\left\|\left(\theta_{d}+\theta_{u}\right)\right\|^{2} \\
\leq & -N_{2}\left(\|\bar{e}\|^{2}+\|\eta\|^{2}\right)+\frac{1}{2}\left\|\left(\theta_{d}+\theta_{u}\right)\right\|^{2} \\
\equiv & -N_{2}\left\|y_{\text {total }}\right\|^{2}+\frac{1}{2}\left\|\left(\theta_{d}+\theta_{u}\right)\right\|^{2},
\end{aligned}
$$

where

$$
\left\|y_{\text {total }}\right\|^{2} \equiv\|\bar{e}\|^{2}+\|\eta\|^{2}
$$

Applying ([29], Theorem 5.2) and (41) implies the input-tostate stability property for the overall system. Furthermore, it is easy to obtain the following inequality:

$$
\omega_{1}\left(\|\bar{e}\|^{2}+\|\eta\|\right) \leq V \leq \omega_{2}\left(\|\bar{e}\|^{2}+\|\eta\|^{2}\right),
$$

that is,

$$
\omega_{1}\left\|y_{\text {total }}\right\|^{2} \leq V \leq \omega_{2}\left\|y_{\text {total }}\right\|^{2}
$$

where $\omega_{1} \equiv \min \left\{\sigma \delta \lambda_{\min }, k_{1}\right\}$ and $\omega_{2} \equiv \min \left\{\sigma \delta \lambda_{\max }, \mu k_{2}\right\}$. Applying (41) and (44) gives

$$
\begin{aligned}
\dot{V} & \leq-\frac{N_{2}}{\omega_{2}} V+\frac{1}{2}\left\|\left(\theta_{d}+\theta_{u}\right)\right\|^{2} \\
& \leq-\frac{N_{2}}{\omega_{2}} V+\frac{1}{2}\left(\sup _{t_{0} \leq \tau \leq t}\left\|\left(\theta_{d}+\theta_{u}\right)(\tau)\right\|\right)^{2} .
\end{aligned}
$$

Hence,

$$
V(t) \leq V\left(t_{0}\right) e^{-\left(N_{2} / \omega_{2}\right)\left(t-t_{0}\right)}+\frac{\omega_{2}}{2 N_{2}}\left(\sup _{t_{0} \leq \tau \leq t}\left\|\left(\theta_{d}+\theta_{u}\right)(\tau)\right\|\right)^{2},
$$

which implies

$$
\begin{aligned}
\left|e_{1}(t)\right| \leq & \sqrt{\frac{V\left(t_{0}\right)}{\sigma \delta \lambda_{\min }}} e^{-\left(N_{2} / 2 \omega_{2}\right)\left(t-t_{0}\right)} \\
& +\sqrt{\frac{\omega_{2}}{2 N_{2} \sigma \delta \lambda_{\min }}}\left(\sup _{t_{0} \leq \tau \leq t}\left\|\left(\theta_{d}+\theta_{u}\right)(\tau)\right\|\right),
\end{aligned}
$$

so that the almost disturbance decoupling property (20b) is achieved. Utilizing (41) yields

$$
\dot{V} \leq-N_{2}\left(\|\bar{e}\|^{2}+\|\eta\|^{2}\right)+\frac{1}{2}\left\|\left(\theta_{d}+\theta_{u}\right)\right\|^{2}
$$

which easily implies

$$
\begin{aligned}
& \int_{t_{0}}^{t}\left(y(\tau)-y_{d}(\tau)\right)^{2} d \tau \\
& \quad \leq \frac{V\left(t_{0}\right)}{N_{2}}+\frac{1}{2 N_{2}} \int_{t_{0}}^{t}\left\|\left(\theta_{d}+\theta_{u}\right)(\tau)\right\|^{2} d \tau,
\end{aligned}
$$

so that the almost disturbance decoupling property (20c) is satisfied and then the tracking problem with almost disturbance decoupling property is globally solved. Finally, we will exploit the fact that the ball $B_{r}$ is a global attractor of system (1a)-(1b). Applying (41) and (23e) gives

$$
\dot{V} \leq-N_{2}\left(\left\|y_{\text {total }}\right\|^{2}\right)+N_{1} \text {. }
$$

For $\left\|y_{\text {total }}\right\|>\underline{r}$, we get $\dot{V}<0$. Hence, any ball denoted as

$$
B_{\underline{r}} \equiv\left\{\left[\begin{array}{l}
\bar{e} \\
\eta
\end{array}\right]:\|\bar{e}\|^{2}+\|\eta\|^{2} \leq \underline{r}\right\}
$$

is a global final attractor of the systems (1a)-(1b). Furthermore, for $y_{\text {total }} \notin B_{\bar{r}}$, we have

$$
\frac{\dot{V}}{V} \leq \frac{-N_{2}}{\omega_{2}}+\frac{N_{1}}{\omega_{2}\left\|y_{\text {total }}\right\|^{2}} \leq \frac{-N_{2}}{\omega_{2}}+\frac{N_{1}}{\omega_{2} \underline{r}^{2}} \equiv-\alpha^{*}
$$

Utilizing the comparison theorem [32] gives

$$
V\left(y_{\text {total }}(t)\right) \leq V\left(y_{\text {total }}\left(t_{0}\right)\right) \exp \left[-\alpha^{*}\left(t-t_{0}\right)\right]
$$

Therefore,

$$
\begin{aligned}
\omega_{1}\left\|y_{\text {total }}\right\|^{2} & \leq V\left(y_{\text {total }}(t)\right) \\
& \leq \omega_{2}\left\|y_{\text {total }}\left(t_{0}\right)\right\|^{2} \exp \left[-\alpha^{*}\left(t-t_{0}\right)\right] .
\end{aligned}
$$

Consequently, we get

$$
\left\|y_{\text {total }}\right\| \leq \sqrt{\frac{\omega_{2}}{\omega_{1}}}\left\|y_{\text {total }}\left(t_{0}\right)\right\| \exp \left[-\frac{1}{2} \alpha^{*}\left(t-t_{0}\right)\right]
$$

that is, the convergence rate toward the ball $B_{r}$ is equal to $\alpha^{*} / 2$. 
TABLE 1: Rule base table of fuzzy control.

\begin{tabular}{|c|c|c|c|c|c|c|c|c|}
\hline \multirow{2}{*}{\multicolumn{2}{|c|}{$u_{\text {fuzzy }}$}} & \multicolumn{7}{|c|}{$e(t)$} \\
\hline & & NB & $\mathrm{NM}$ & NS & $\mathrm{ZE}$ & PS & $\mathrm{PM}$ & $\mathrm{PB}$ \\
\hline \multirow{7}{*}{$\dot{e}(t)$} & NB & $\mathrm{PB}$ & $\mathrm{PB}$ & $\mathrm{PB}$ & $\mathrm{PB}$ & $\mathrm{PM}$ & PS & $\mathrm{ZE}$ \\
\hline & NM & $\mathrm{PB}$ & $\mathrm{PB}$ & $\mathrm{PB}$ & $\mathrm{PM}$ & PS & $\mathrm{ZE}$ & NS \\
\hline & NS & $\mathrm{PB}$ & PB & $\mathrm{PM}$ & PS & $\mathrm{ZE}$ & NS & NM \\
\hline & ZE & $\mathrm{PB}$ & $\mathrm{PM}$ & PS & $\mathrm{ZE}$ & NS & $\mathrm{NM}$ & NB \\
\hline & PS & PM & PS & $\mathrm{ZE}$ & NS & $\mathrm{NM}$ & NB & NB \\
\hline & $\mathrm{PM}$ & PS & $\mathrm{ZE}$ & NS & $\mathrm{NM}$ & $\mathrm{NB}$ & NB & NB \\
\hline & $\mathrm{PB}$ & $\mathrm{ZE}$ & NS & NM & $\mathrm{NB}$ & $\mathrm{NB}$ & NB & NB \\
\hline
\end{tabular}

2.2. Controller Design for Fuzzy Control Approach. After applying feedback linearization controller as a guarantee of uniform ultimate bounded stability, the fuzzy controller can be significantly utilized to obtain the better convergence rate of tracking error dynamics. The block diagram of the fuzzy controller is given in Figure 1. In general, the tracking error $e(t)$ and its time derivative $\dot{e}(t)$ are applied as the input fuzzy variables of the IF-THEN control rules and the output variable is the control $u_{\text {fuzzy }}$.

For the sake of simplification, the membership functions of the linguistic terms for $e(t), \dot{e}(t)$, and $u_{\text {fuzzy }}$ are all expressed to be the triangular functions. We denote seven linguistic terms for each fuzzy variable as shown in Figure 2: PB (positive big), PM (positive medium), PS (positive small), ZE (zero), NS (negative small), NM (negative medium) and NB (negative big).

Rule base table of fuzzy control $u_{\text {fuzzy }}$ is shown in Table 1. The rule base is constructed by the Macvicar-Whelan rule base [33] for general servo control systems. The Mamdani method [34] is utilized for fuzzy inference. The defuzzification of the output set membership value is constructed by the centroid approach. Therefore, we can integrate the feedback linearization control and fuzzy control to build the controller as follows:

$$
\begin{aligned}
u_{f e+f u} \equiv & u_{\text {feedback }} u_{s}(t)+u_{\text {fuzzy }} u_{s}\left(t-t_{1}\right) \\
= & {\left[L_{g} L_{f}^{r-1} h(X(t))\right]^{-1} } \\
& \times\left\{-L_{f}^{r} h(X)+y_{t}^{(r)}-\sigma^{-r} n_{1}\left[L_{f}^{0} h(X)-y_{t}\right]\right. \\
& -\sigma^{1-r} n_{2}\left[L_{f}^{1} h(X)-y_{t}^{(1)}\right] \\
& \left.-\cdots-\sigma^{-1} n_{r}\left[L_{f}^{r-1} h(X)-y_{t}^{(r-1)}\right]\right\} u_{s}(t) \\
& +u_{\text {fuzzy }} u_{s}\left(t-t_{1}\right)
\end{aligned}
$$

where $u_{s}$ is the unit step function and $t_{1}$ is the time that the tracking error dynamics touch the final global attractor ball $B_{\underline{r}}$.

\section{Unmanned Underwater Vehicle}

The coordinate system of an unmanned underwater vehicle with one propeller, two stern planes, and two rudders is shown in Figure 3. The six degree-of-freedom nonlinear dynamics of the UUV control system can be described as follows [5]:

$$
\begin{gathered}
M(\nu) \dot{v}+C_{D}(\nu) \nu+D(\nu) \nu+g(\eta)+d=\tau, \\
\dot{\eta}=J(\eta),
\end{gathered}
$$

where $\eta=\left[\begin{array}{llllll}x & y & z & \phi & \theta & \psi\end{array}\right]^{T}$ denotes the position and orientation vector in earth-fixed reference frame, $v=$ $\left[\begin{array}{llllll}u & v & w & p & q & r\end{array}\right]^{T}$ denotes the velocity and angular rate vector in vehicle (body)-fixed reference frame, $M(\nu) \in R^{6 \times 6}$ denotes the inertia matrix including rigid-body mass and added mass, $C_{D}(\nu) \in R^{6 \times 6}$ denotes the matrix of Coriolis, centripetal term, $D(\nu) \in R^{6 \times 6}$ denotes the damping matrix, $g(\eta) \in R^{6}$ denotes the gravitational forces and moments vector, $d$ denotes the exogenous disturbance term, $\tau$ is the torque input vector, and $J(\eta)$ denotes the transformation matrix [5]. The UUV is considered to be longitudinal and bilateral symmetry and it is reasonable to make the assumption that the origin of the body-fixed coordinate is located at the center of gravity, and the terms in $g(\eta) \in R^{6}$ is all zero. For the dynamics of the diving plane, we can assume that the roll and yaw angular velocities are close to zeros based on appropriately adjusting the RPM of propeller and the rudder's angles. For the discussion of the horizontal moving dynamics in this study, the forward velocity is kept constant $u_{0}$ by properly adjusting the rev of the thrusters. Under the above assumptions, the heave dynamics of UUV can be described as

$$
\dot{z}=-u \sin \theta+v \cos \theta \sin \phi+\omega \cos \theta \cos \phi \approx-u_{0} \sin \theta .
$$

The roll angle $\phi$ is nearly constant, since $p \approx 0$. Without any loss of generality, we can assume $\phi=0$ and the pitch dynamics can be expressed as

$$
\dot{\theta}=q \cos \phi-r \sin \phi \approx q \cos \phi \approx q .
$$

Consequently, the diving dynamics of UUV can be certain modified as followed [5-7]:

$$
\begin{gathered}
\dot{z}=-u_{0} \sin \theta, \\
\dot{\theta}=q, \\
m_{q} \dot{q}=\Phi^{T} \Theta+F_{q} u_{0}^{2} \delta_{q}+d_{q},
\end{gathered}
$$

where

$$
\begin{gathered}
\Phi=\left[\begin{array}{llllllll}
q & \dot{u} & u & u^{2} & \omega q & r q & \cos \phi \sin \Psi
\end{array}\right]^{T}, \\
\Theta=\left[\begin{array}{llllllll}
\theta_{1} & \theta_{2} & \theta_{3} & \theta_{4} & \theta_{5} & \theta_{6} & \theta_{7}
\end{array}\right]^{T},
\end{gathered}
$$

and $m_{q}$ denotes the inertia term including the added mass, $F_{q}$ denotes the fin moment coefficient, and $\delta_{q}$ denotes the stern plane angle as depicted in Figure 4.

Substituting all the physical values $m_{q}=3.495, F_{q}=$ 6.51, $u_{0}=1.54$, and selecting $x_{1} \equiv z, x_{2} \equiv \theta, x_{3} \equiv q$, 


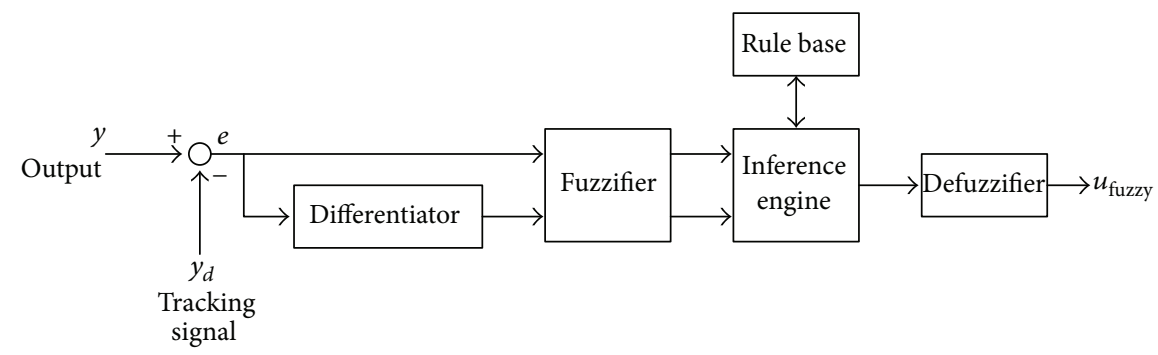

FIGURE 1: Block diagram of fuzzy logic controller.

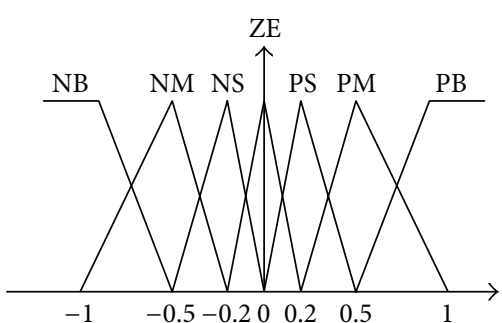

(a) $e(t)$

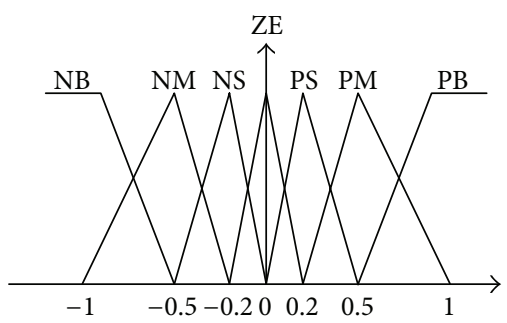

(b) $\dot{e}(t)$

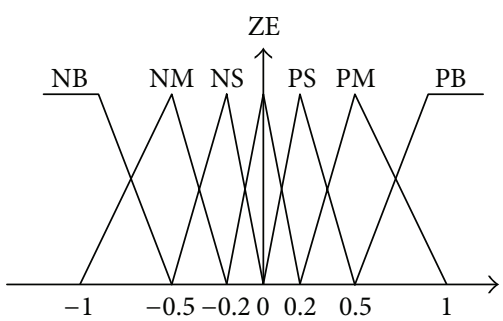

(c) $u_{\text {fuzzy }}$

Figure 2: Plots of membership functions for (a) $e(t)$, (b) $\dot{e}(t)$, and (c) $u_{\text {fuzzy }}$.

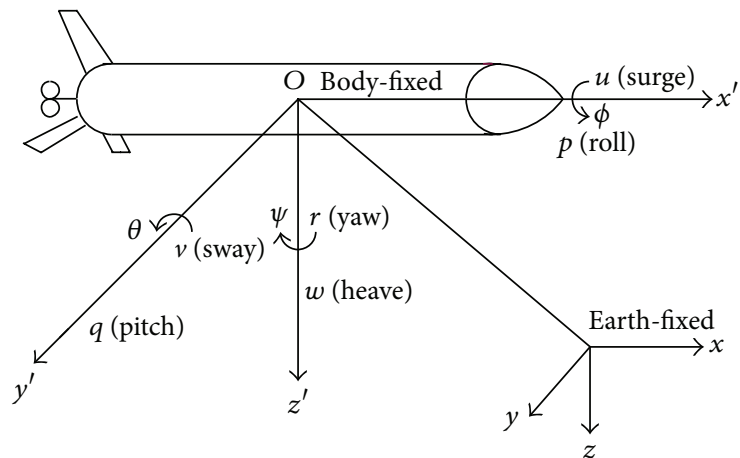

FIGURE 3: The unmanned underwater vehicle.

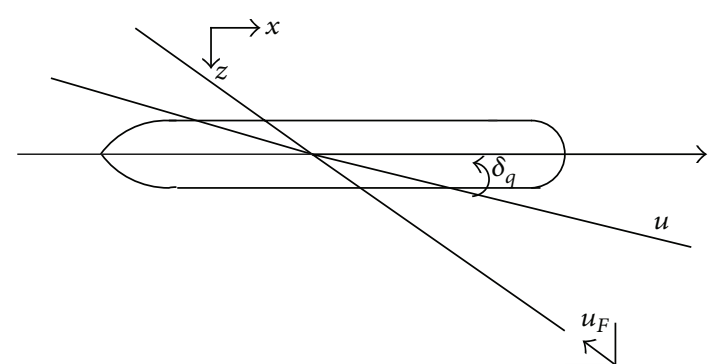

FIGURE 4: The stern plane angle of attack.

$\theta_{1}=1, \theta_{7}=1$ into (60a), (60b), (60c), (60d), and (60e), the state equation can be rewritten as follows:

$$
\begin{gathered}
\dot{x}_{1}=-1.54 \sin x_{2}, \\
\dot{x}_{2}=x_{3}, \\
\dot{x}_{3}=\frac{1}{3.495} x_{3}+\frac{1}{3.495} \sin x_{2}+15.5126 u+d_{q},
\end{gathered}
$$

where $d_{r}=0.5 \sin t$ is assumed to be the disturbance item. For a given desired trajectory $y_{t}=\sin 0.1 t$, the main objective of this study is to construct a nonlinear feedback linearization controller for UUV system (61a), (61b), and (61c) with tracking performance by applying Theorem 4 . Let us arbitrarily choose $n_{1}=1.5, n_{2}=1.5, n_{3}=1.5$ such that

$$
A_{\mathrm{pvc}}=\left[\begin{array}{ccc}
0 & 1 & 0 \\
0 & 0 & 1 \\
-1.5 & -1.5 & -1.5
\end{array}\right]
$$

is Hurwitze and the positive definite matrix

$$
P_{\mathrm{pd}}=\left[\begin{array}{ccc}
3.5 & 2.5 & 0.25 \\
2.5 & 4.75 & 1.5 \\
0.25 & 1.5 & 1
\end{array}\right]
$$

The UUV system is a system of relative degree three. It can be verified that the related conditions of Theorem 4 are satisfied 


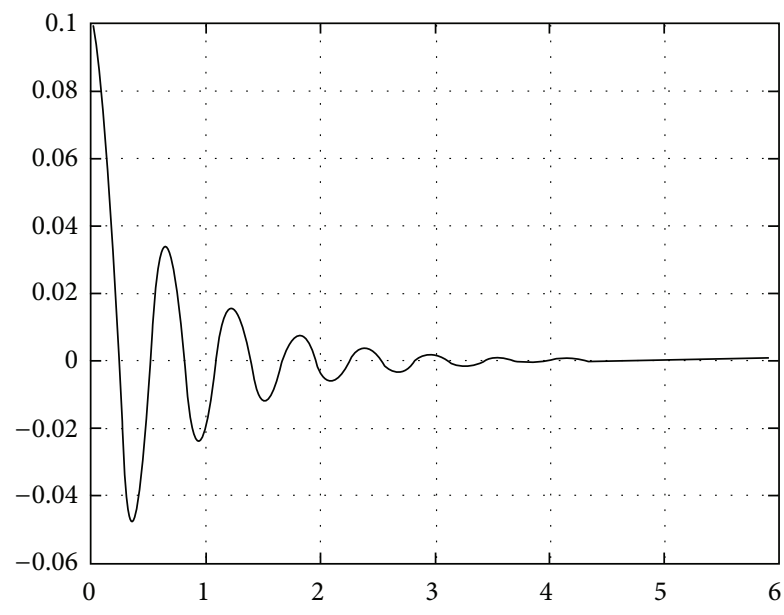

FIGURE 5: The tracking error dynamic $x_{1}-\sin 0.1 t$ driven by $u$ for (61a), (61b), and (61c).

with $\sigma=0.1, m_{11}=1.75, \underline{r}=0.4, N_{1}=0.125$, and $N_{2}=$ 1.75. From (56), we obtain the desired tracking controller

$$
\begin{gathered}
u=\left[L_{g} L_{f}^{2} h(X(t))\right]^{-1} \\
\times\left\{-L_{f}^{3} h(X)+y_{t}^{(3)}-\sigma^{-3} n_{1}\left[h(X)-y_{t}\right]\right. \\
-\sigma^{-2} n_{2}\left[L_{f}^{1} h(X)-y_{t}^{(1)}\right] \\
\left.-\sigma^{-1} n_{3}\left[L_{f}^{2} h(X)-y_{t}^{(2)}\right]\right\} u_{s}(t) \\
+u_{\text {fuzzy }} u_{s}\left(t-t_{1}\right) \\
L_{g} L_{f}^{2} h(X)=-23.889 \cos x_{2}, \\
L_{f}^{3} h(X)=1.54 x_{3}^{2} \sin x_{2} \\
-1.54 \cos x_{2}\left(\frac{k_{1}}{3.495} x_{3}+\frac{k_{7}}{3.495} \sin x_{2}\right) \\
{ }^{2}{ }_{f}^{(3)} h(X)=-0.001 \cos 0.1 t \\
L_{f}^{1} h(X)=-1.54 \sin x_{2} \\
y_{d}^{(1)}=0.1 \cos 0.1 t \\
\left(64 x_{3} \cos x_{2} .\right.
\end{gathered}
$$

Hence, the tracking controller will steer the state variable $x_{1}$ to track the desired trajectory $y_{t}(t)=h(X)=\sin 0.1 t$ in view of Theorem 4 . The tracking errors driven by $u$ for UUV system (61a), (61b), and (61c) is depicted in Figure 5.

\section{Conclusion}

In this paper, we have proposed a fuzzy feedback control design which globally solves the almost disturbance decoupling problem based on the fuzzy feedback linearization approach for trajectory tracking using a rudder of a UUV. The investigation of input-output feedback linearization of nonlinear uncertain control systems by diffeomorphism has been proposed without eliminating the nonlinear terms in the UUV model, and so severe model errors will not be taken into the developing process. Moreover, this study proposes a control algorithm for diving dynamics of UUV without restricting constraint on the small pitch angle in the diving plane. Simulation results exploit the fact that our proposed design is favorably applied to input-output linearization problem and achieves the desired almost disturbance decoupling performance and better convergence rate of the closedloop system.

\section{References}

[1] A. Faruq, S. S. Abdullah, M. Fauzi, and S. Nor, "Optimization of depth control for Unmanned Underwater Vehicle using surrogate modeling technique," in Proceedings of the 4th International Conference on Modeling, Simulation and Applied Optimization (ICMSAO '11), pp. 1-7, Kuala Lumpur, Malaysia, April 2011.

[2] T. Lugaric, A. Vasilijevic, and S. Srbljic, "Interconnectable gadgets and web services usage in supervisory control of Unmanned Underwater vehicles," in Proceedings of the 34th International Convention MIPRO, pp. 696-701, Opatija, Croatia, 2011.

[3] J. J. E. Slotine and W. Li, Applied Nonlinear Control, PrenticeHall, New York, NY, USA, 1991.

[4] A. J. Healey and D. Lienard, "Multivariable sliding mode control for autonomous diving and steering of unmanned underwater vehicles," IEEE Journal of Oceanic Engineering, vol. 18, no. 3, pp. 327-339, 1993.

[5] T. I. Fossen, Guidance and Control of Ocean Vehicles, John Wiley \& Sons, Chichester, UK, 1994.

[6] T. Prestero, Verification of a six-degree of freedom simulation model for the REMUS autonomous underwater vehicles [M.S. thesis], Department of Ocean Engineering and Mechanical Engineering, The MIT Press, Cambridge, Mass, USA, 2001.

[7] J. H. Li, P. M. Lee, and B. H. Jun, "An adaptive nonlinear controller for diving motion of an UUV," in Proceedings of the MTTS/IEEE Techno-Ocean (Oceans '04), vol. 1, pp. 282-287, November 2004.

[8] M. Morawiec, "The adaptive backstepping control of permanent magnet synchronous motor supplied by current source inverter," IEEE Transactions on Industrial Informatics, vol. 9, no. 2, pp. 1047-1055, 2013.

[9] M. Krstic, I. Kanellakopoulos, and P. V. Kokotovic, Nonlinear and Adaptive Control Design, John Wiley \& Sons, New York, NY, USA, 1995.

[10] S. Y. Ou, C. Y. Tang, and Z. J. Chen, "Design and implementation of a ZCS-PWM half-bridge boost rectifier with output voltage balance control," IEEE Transactions on Industrial Electronics, vol. 59, no. 12, pp. 4646-4656, 2012.

[11] P. Zhaowu, "Approximate output regulation of spherical inverted pendulum by neural network enhanced design," in 
Proceedings of the 31st Chinese Control Conference, pp. 361-366, 2012.

[12] C. G. Claudel, T. Chamoin, and A. M. Bayen, "Solutions to estimation problems for Scalar Hamilton-Jacobi equations using linear programming," IEEE Transactions on Control Systems Technology, no. 99, 1 pages, 2013.

[13] N. O. Pérez-Arancibia, J. P. Whitney, and R. J. Wood, "Lift force control of flapping-wing microrobots using adaptive feedforward schemes," IEEE/ASME Transactions on Mechatronics, vol. 18, no. 1, pp. 155-168, 2013.

[14] S. Galeani, L. Menini, and A. Potini, "Robust trajectory tracking for a class of hybrid systems: an internal model principle approach," IEEE Transactions on Automatic Control, vol. 57, no. 2, pp. 344-359, 2012.

[15] V. Azimi, A. Vahid, and M. H. Kazemi, "Robust multi objective $\mathrm{H} 2 / \mathrm{H} \infty$ control of nonlinear uncertain systems using multiple linear model and ANFIS," in Proceedings of the Chinese Control and Decision Conference, pp. 2641-2646, 2011.

[16] T. L. Chien, C. C. Chen, M. C. Tsai, and Y. C. Chen, "Control of AMIRA's ball and beam system via improved fuzzy feedback linearization approach," Applied Mathematical Modelling, vol. 34, no. 12, pp. 3791-3804, 2010.

[17] A. Hamadi, S. Rahmani, and K. Al-Haddad, "Digital control of a shunt hybrid power filter adopting a nonlinear control approach," IEEE Transactions on Industrial Informatics, no. 99, 1 pages, 2013.

[18] X. Bao, F. Zhuo, Y. Tian, and P. Tan, "Simplified feedback linearization control of three-phase photovoltaic inverter with an LCL filter," IEEE Transactions on Power Electronics, vol. 28, no. 6, pp. 2739-2752, 2013.

[19] Z. Li, X. Cao, Y. Tian, R. Li, and W. Ye, "Bilateral teleoperation of holonomic constrained robotic systems with time-varying delays," IEEE Transactions on Instrumentation and Measurement, vol. 62, no. 4, pp. 752-765, 2013.

[20] K. Noori and K. Jenab, "Fuzzy reliability-based traction control model for intelligent transportation systems," IEEE Transactions on Systems, Man, and Cybernetics, vol. 43, no. 1, pp. 229-234, 2013.

[21] X. Xie, H. Ma, D. W. Ding, and Y. Wang, "Control synthesis of discrete-time T-S fuzzy systems based on a novel non-PDC control scheme," IEEE Transaction on Fuzzy Systems, vol. 21, no. 1, pp. 147-157, 2013.

[22] S. Elbeid and S. Doubabi, "DSP-based implementation of fuzzy output tracking control for a boost converter," IEEE Transactions on Industrial Electronics, no. 99, 1 pages, 2013.

[23] J. Zhang, P. Shi, and Y. Xia, "Fuzzy delay compensation control for T-S fuzzy systems over network," IEEE Transactions on Cybernetics, vol. 43, no. 1, pp. 259-268, 2013.

[24] C. S. S. Chiu and Y. L. Ouyang, "Robust mximum power tracking control of uncertain photovoltaic systems: a unified TS fuzzy model-based approach," IEEE Transactions on Control Systems Technology, vol. 19, no. 6, pp. 1516-1526, 2011.

[25] M. C. Jesús Alberto, G. M. Julio César, and C. T. Bernardino, "Exact output regulation for nonlinear systems described by Takagi-Sugeno fuzzy models," IEEE Transactions on Fuzzy Systems, vol. 20, no. 2, pp. 235-247, 2012.

[26] Y. Huang, D. P. Palomar, and S. Zhang, "Lorentz-positive maps and quadratic matrix inequalities with applications to robust MISO transmit beamforming," IEEE Transactions on Cybernetics, vol. 61, no. 5, pp. 1121-1130, 2013.
[27] A. Levant, "Practical relative degree in black-box control," in Proceedings of the IEEE 51st Annual Conference on Decision and Control, pp. 7101-7106, 2012.

[28] Z. Yang, S. Hua, Q. Hongzhuan, and L. Chengrui, "Control reconfigurability of nonlinear system based on control redundancy," in Proceedings of the 10th IEEE International Conference on Industrial Informatics, pp. 815-820, 2012.

[29] H. K. Khali, Nonlinear Systems, Prentice-Hall, New Jersey, NJ, USA, 1996.

[30] R. Marino and P. Tomei, "Nonlinear output feedback tracking with almost disturbance decoupling," IEEE Transactions on Automatic Control, vol. 44, no. 1, pp. 18-28, 1999.

[31] J. Anderson and A. Papachristodoulou, "A decomposition technique for nonlinear dynamical system analysis," IEEE Transactions on Automatic Control, vol. 57, no. 6, pp. 1516-1521, 2012.

[32] W. Zhang and J. Hu, "An ODE comparison theorem with application in the optimal exit time control problem," IEEE Transactions on Automatic Control, vol. 55, no. 1, pp. 164-170, 2010.

[33] M. Biglarbegian, W. W. Melek, and J. M. Mendel, "A practical approach for design of PD and PI like interval type-2 TSK fuzzy controllers," in Proceedings of the IEEE International Conference on Systems, Man and Cybernetics (SMC'09), pp. 255261, October 2009.

[34] N. Wang, C. Hu, and W. Shi, "A mamdani fuzzy modeling method via evolution-objective cluster analysis," in Proceedings of the 31st Chinese Control Conference, pp. 3470-3475, 2012. 


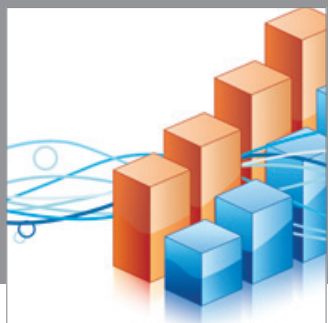

Advances in

Operations Research

mansans

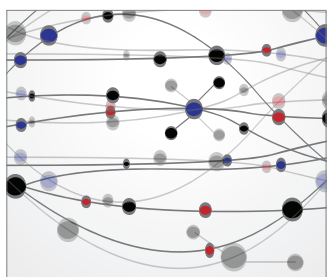

The Scientific World Journal
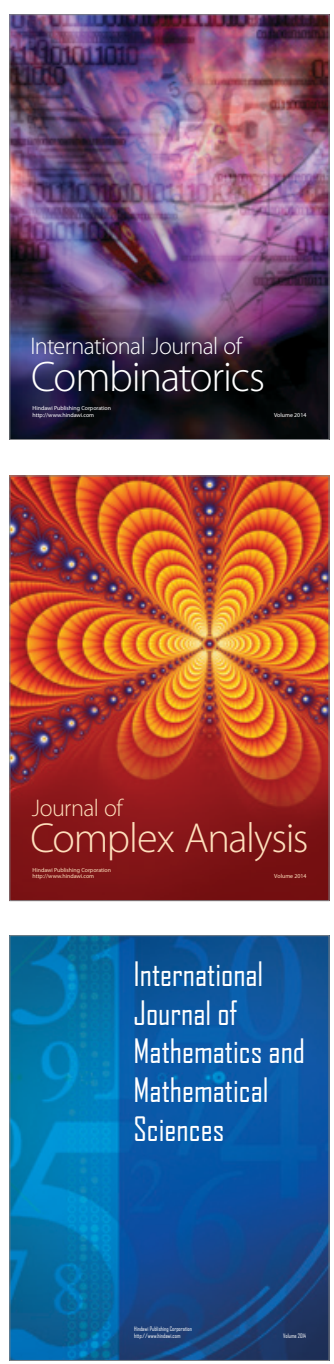
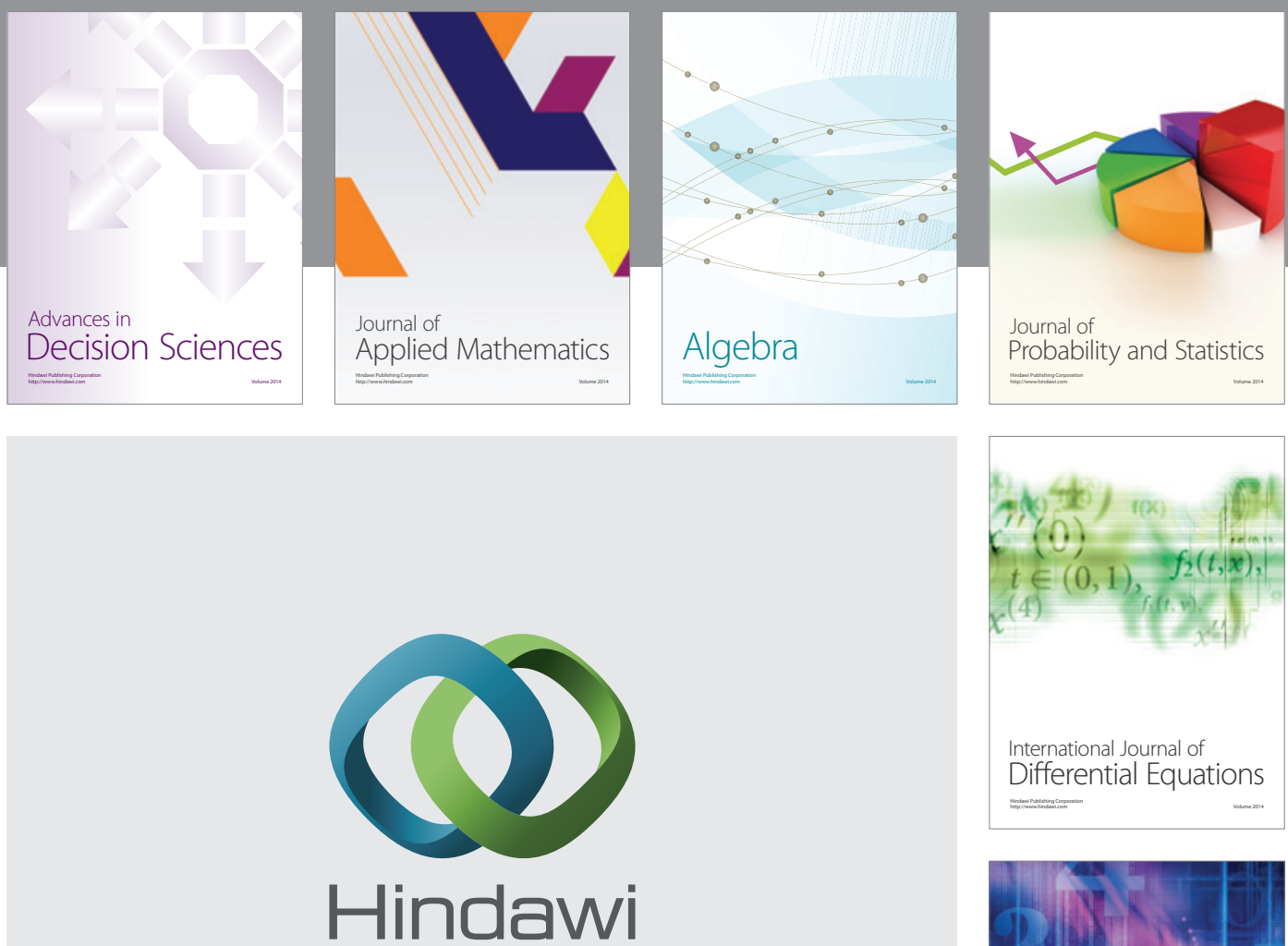

Submit your manuscripts at http://www.hindawi.com
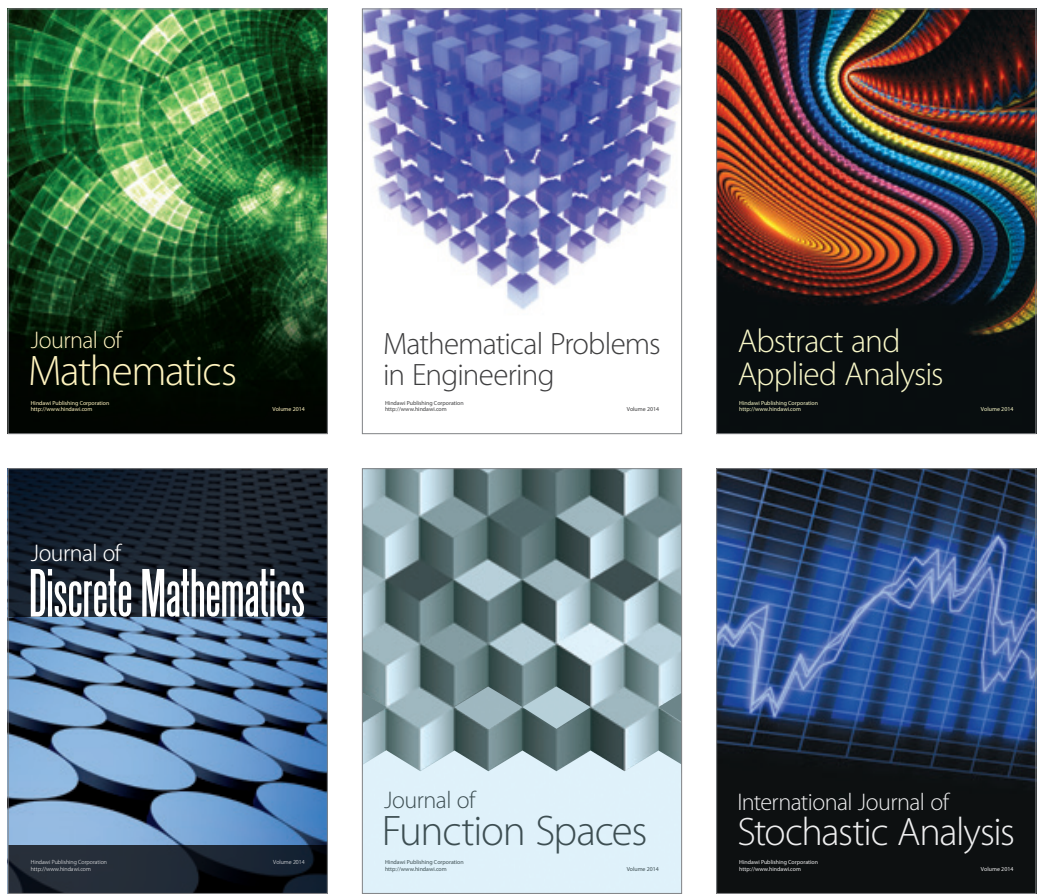

Journal of

Function Spaces

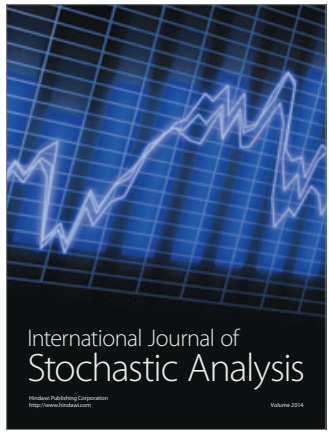

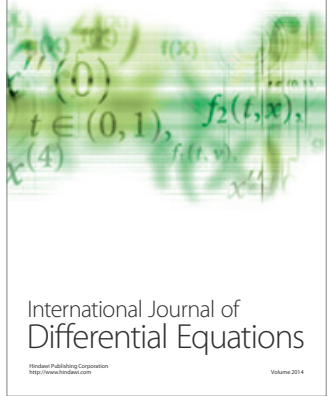
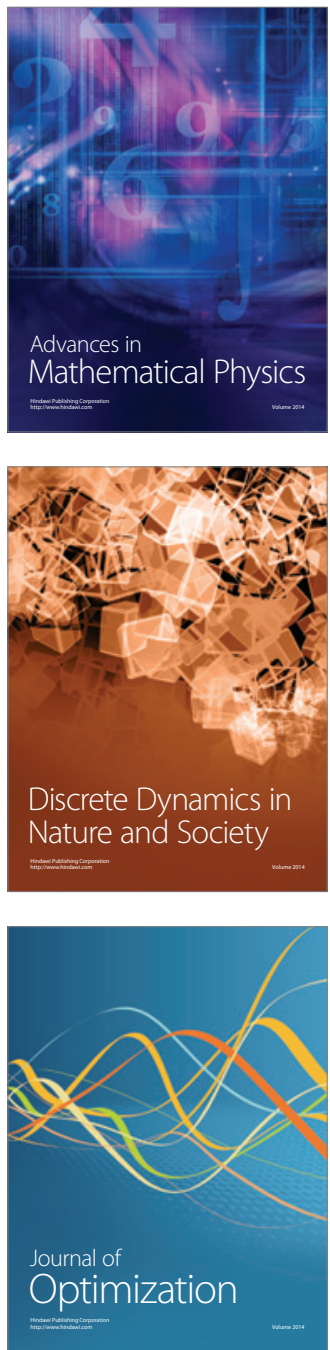